\title{
Trailing end-point phenotype antibiotic-sensitive strains of Candida albicans produce different amounts of aspartyl peptidases
}

\author{
L.A. Braga-Silva ${ }^{1,3}$, D.G.A. Mesquita ${ }^{1}$, M.D. Ribeiro ${ }^{2,4}$, \\ S.M.F. Carvalho ${ }^{4}$, S.E.L. Fracalanzza ${ }^{3}$ and A.L.S. Santos ${ }^{1,3}$ \\ ${ }^{1}$ Laboratório de Estudos Integrados em Bioquímica Microbiana, Departamento de Microbiologia Geral, \\ 2Laboratório de Bacteriologia Médica, Departamento de Microbiologia Médica, Instituto de Microbiologia \\ Prof. Paulo de Góes, Universidade Federal do Rio de Janeiro, Rio de Janeiro, RJ, Brasil \\ ${ }^{3}$ Programa de Pós-Graduação em Bioquímica, Instituto de Química, Universidade Federal \\ do Rio de Janeiro, Rio de Janeiro, RJ, Brasil \\ ${ }^{4}$ Laboratório de Microbiologia (Micologia), Instituto Estadual de Hematologia \\ Arthur de Siqueira Cavalcanti, HEMORIO, Rio de Janeiro, RJ, Brasil
}

\begin{abstract}
Candida albicans is an opportunistic fungal pathogen that causes severe systemic infections in immunosuppressed individuals. C. albicans resistance to antifungal drugs is a severe problem in patients receiving prolonged therapy. Moreover, trailing yeast growth, which is defined as a resistant MIC after $48 \mathrm{~h}$ of incubation with triazole antifungal agents but a susceptible MIC after $24 \mathrm{~h}$, has been noted in tests of antifungal susceptibility against some $C$. albicans isolates. In this context, we recently noticed this phenomenon in our routine susceptibility tests with fluconazole/itraconazole and $C$. albicans clinical isolates. In the present study, we investigated the production of cell-associated and secreted aspartyl peptidases (Saps) in six trailing clinical isolates of $C$. albicans, since this class of hydrolytic enzymes is a well-known virulence factor expressed by this fungal pathogen. Sap2, which is the best-studied member of the Sap family, was detected by flow cytometry on the cell surface of yeasts and as a 43-kDa polypeptide in the culture supernatant, as demonstrated by Western blotting assay using an anti-Sap1-3 polyclonal antibody. Released aspartyl peptidase activity was measured with BSA hydrolysis and inhibited by pepstatin A, showing distinct amounts of proteolytic activity ranging from 5.7 (strain 44B) to 133.2 (strain 11) arbitrary units. Taken together, our results showed that trailing clinical isolates of $C$. albicans produced different amounts of both cellular and secreted aspartyl-type peptidases, suggesting that this phenotypic feature did not generate a regular pattern regarding the expression of Sap.
\end{abstract}

Key words: Candida albicans; Trailing growth; Secreted aspartyl peptidases; Sap2

\section{Introduction}

Candida albicans is commonly found on the mucosal surfaces of the gastrointestinal and genitourinary tracts in many healthy people. However, it can also cause opportunistic infections, especially in immunocompromised, cancer and transplanted patients $(1,2)$. C. albicans possesses a number of virulence attributes that enable the organism to cause disseminated infections in susceptible hosts and that also favor the persistence and colonization of the host tissues. Putative virulence factors of $C$. albicans include cell wall adhesins, phenotypic switching, hypha formation, thigmotropism, and secretion of hydrolytic enzymes, such as phospholipases and proteolytic enzymes $(3,4)$. Among these virulence factors, enzymes with proteolytic activity continue to attract the attention of many investigators who study the pathogenesis of candidiasis. Secreted aspartyl peptidases (Saps), encoded by the Sap gene family with

Correspondence: A.L.S. Santos, Laboratório de Estudos Integrados em Bioquímica Microbiana, Departamento de Microbiologia Geral, Instituto de Microbiologia Prof. Paulo de Góes, Bloco E-subsolo, CCS, UFRJ, Av. Carlos Chagas Filho, 373, 21941-902 Rio de Janeiro, RJ, Brasil. E-mail: andre@micro.ufrj.br or alsouzasantos@gmail.com

Research supported by CNPq, FAPERJ, and Fundação Universitária José Bonifácio (FUJB). L.A. Braga-Silva, a postgraduate student of Programa de Pós-graduação em Bioquímica, Instituto de Química, Centro de Tecnologia, Universidade Federal do Rio de Janeiro, was supported by a CNPq fellowship.

Received October 26, 2008. Accepted July 6, 2009. 
10 members (Sap1 to Sap10), appear to play a major role in $C$. albicans virulence during fungal infections (reviewed in Ref. 4).

The infections caused by Candida spp result in increased length of hospital stay and medical costs, which constitute an important public health problem (5). Fluconazole and other antifungal azoles have proved to be effective for the treatment of mucosal and systemic Candida infections; however, they lack fungicidal activity and treatment failures are common in severely compromised patients. Such failures have been associated with the emergence of azole-resistant strains of $C$. albicans during treatment, and resistance has been shown to be a direct consequence of the daily cumulative doses of azoles (6-8). In vitro, azoles not only fail to kill but, in a number of clinical isolates, also fail to truly suppress growth, resulting in trailing growth in broth microdilution assays. Among fungal pathogens, in vivo and in vitro acquired resistance to azoles and other drugs has been shown to be inducible and rapid but transient, and has not been associated with plasmids or other transferable genetic elements, but is thought to involve primarily mutations and genetic or epigenetic rearrangements $(9,10)$. Interestingly, a positive correlation of peptidase activity with antifungal susceptibility was demonstrated in $C$. albicans. For example, C. albicans isolates from the HIV-positive group that were characterized by higher levels of aspartyl-type proteolytic activity were also less susceptible to the widely used azole antifungal agents ketoconazole and fluconazole (11). It has been thought that the proteolytic activity increases resistance to azole group antifungal agents especially in immunosuppressed patients (12).

Recently, Ribeiro (13) noticed the trailing phenomenon in routine susceptibility tests with fluconazole/itraconazole and C. albicans isolated from different hospitals from Rio de Janeiro, Brazil. In the present study, we report the measurement of cell-associated and released aspartyltype peptidases in six clinical isolates of $C$. albicans that presented the trailing phenomenon in terms of fluconazole and itraconazole susceptibility in vitro.

\section{Material and Methods}

\section{Candida albicans strains}

The following $C$. albicans strains were employed in this study: C. albicans ATCC strain (code number 36802) and six clinical isolates (Table 1) from patients who were attended at the Laboratório de Microbiologia e Micologia, Instituto Estadual de Hematologia Arthur de Siqueira Cavalcanti (HemoRio), Rio de Janeiro, Brazil (13). In order to minimize the genetic changes over time, each strain was frozen in glycerol and stored at $-80^{\circ} \mathrm{C}$. These stocks were used for all experiments.

\section{Broth microdilution susceptibility testing method}

The fungi were cultured on Sabouraud-dextrose (Oxoid,
England) agar plates for $48 \mathrm{~h}$ at $37^{\circ} \mathrm{C}$. The minimum inhibitory concentrations (MICs) of fluconazole and itraconazole for the $C$. albicans isolates were determined by the microdilution broth method (14) according to the guidelines of the Clinical and Laboratory Standards Institute (formerly National Committee for Clinical Laboratory Standards) (15), document M27-A. The MIC was read spectrophotometrically and the end point calculated as a $50 \%$ reduction in absorbance as compared to the growth in the control well. Trailing growth was defined as a resistant MIC after $48 \mathrm{~h}$ of incubation but a susceptible MIC after $24 \mathrm{~h}$ of incubation.

\section{Culture conditions and evaluation of cell growth}

C. albicans strains were inoculated into $1.2 \%(\mathrm{w} / \mathrm{v})$ yeast carbon base (YCB) medium (HiMedia Laboratories Ltd., India) supplemented with $0.1 \%(\mathrm{w} / \mathrm{v})$ bovine serum albumin (BSA), $\mathrm{pH} 5.0$, and grown at $37^{\circ} \mathrm{C}$ for $48 \mathrm{~h}$ under slight agitation (100 rpm), which is a well-known Sap-inducing condition $(16,17)$. Cell growth was estimated by counting the yeast cells in a Neubauer chamber.

\section{Yeast and cell-free culture supernatants}

The cultures were harvested by centrifugation at $4000 \mathrm{~g}$ for $10 \mathrm{~min}$ at $4^{\circ} \mathrm{C}$ and the spent culture media were filtered through a 0.22- $\mu \mathrm{m}$ membrane (Millipore, Brazil) and used to measure the extracellular peptidase activity. Alternatively, each cell-free culture supernatant was concentrated 25 -fold in a 10,000-molecular weight cut-off Centricon micropartition system (AMICON, USA) to be employed in Western blotting assays. The same volume of YCB-BSA medium was also concentrated and used as a control (17). Conversely, yeast cells were washed twice in cold phosphate-buffered saline (PBS, $150 \mathrm{mM} \mathrm{NaCl}, 20 \mathrm{mM}$ sodium phosphate buffer, $\mathrm{pH}$ 7.2) and immediately processed by flow cytometry analysis as described below.

\section{Protein profile}

Cell-free culture supernatants of $C$. albicans strains were treated with an equal volume of SDS-PAGE sample buffer (125 mM Tris, pH 6.8, 4\% SDS, 20\% glycerol, and 0.002\% bromophenol blue) containing 10\% $\beta$-mercaptoethanol, followed by heating at $100^{\circ} \mathrm{C}$ for $5 \mathrm{~min}$. Proteins $(10 \mu \mathrm{g}$ of each sample) were analyzed on $10 \%$ SDS-PAGE by the method described by Laemmli (18). Electrophoresis was carried out at $100 \mathrm{~V}$ for $90 \mathrm{~min}$ at $4^{\circ} \mathrm{C}$, and the gels were silver-stained. Prior to electrophoresis, Gibco BRL (USA) molecular mass standards were boiled in SDS-PAGE sample buffer and then applied to the same gel.

\section{Quantitative proteolytic activity assay measured with} BSA

Proteolytic activity was measured spectrophotometrically using BSA as substrate, according to the method described by Buroker-Kilgore and Wang (19) and modified by Silva et al. (20). Initially, each spent culture medium 
was incubated for $20 \mathrm{~min}$ at $37^{\circ} \mathrm{C}$ in the presence or in the absence of $10 \mu \mathrm{M}$ pepstatin A (Sigma, USA), an aspartyl peptidase inhibitor. To assay the remaining proteolytic activity, BSA ( $0.5 \mathrm{mg} / \mathrm{mL}), 20 \mathrm{mM}$ sodium citrate, $\mathrm{pH} 4.0$, and $40 \mu \mathrm{L}$ of each culture supernatant were added to a microcentrifuge tube $(350 \mu \mathrm{L})$ and incubated for $2 \mathrm{~h}$ at $37^{\circ} \mathrm{C}$. After this incubation, three aliquots $(100 \mu \mathrm{L}$ each) of the reaction mixture were transferred to wells on a microtiter plate containing $50 \mu \mathrm{L}$ of water and $100 \mu \mathrm{L}$ of a Coomassie solution $(0.025 \%$ Coomassie brilliant blue G-250, 11.75\% ethanol, and $21.25 \%$ phosphoric acid). After 10 min to allow dye binding, the plate was read on a Thermomax Molecular Device microplate reader at an absorbance of $595 \mathrm{~nm}$. One unit of proteolytic activity was defined as the amount of enzyme that caused an increase of 0.001 in the absorbance under standard assay conditions. The proteolytic activity is expressed as arbitrary units (20).

\section{Immunoblotting assay}

The extracellular polypeptides present in the concentrated supernatant of each C. albicans strain were separated by $10 \%$ SDS-PAGE and then electrophoretically transferred at $4^{\circ} \mathrm{C}$ at $100 \mathrm{~V} / 300 \mathrm{~mA}$ for $2 \mathrm{~h}$ to a nitrocellulose membrane. The membrane was blocked in $5 \%$ low-fat dried milk in TBS (150 mM NaCl, $10 \mathrm{mM}$ Tris, $\mathrm{pH} 7.4$ ) containing 0.5\% Tween 20 (TBS/Tween) for $1 \mathrm{~h}$ at $25^{\circ} \mathrm{C}$. The membrane was washed three times (10 min each) with TBS/Tween and then incubated with the anti-Sap1-3 polyclonal antibody raised against $C$. albicans (kindly provided by Dr. Nina Agabian, University of California, San Francisco, CA, USA) at 1:1000 dilution for $1 \mathrm{~h}(16)$. The secondary antibody used was peroxidase-conjugated goat anti-rabbit $\lg G$ at 1:5000. Immunoblots were exposed to $X$-ray film after reaction with ECL reagents for chemiluminescence (17).

\section{Flow cytometry analysis}

The yeasts $\left(5.0 \times 10^{6}\right.$ cells $)$ used for these experiments were fixed at $4^{\circ} \mathrm{C}$ in $4 \%$ paraformaldehyde in $\mathrm{PBS}, \mathrm{pH} 7.2$, for $20 \mathrm{~min}$, followed by extensive washing in the same buffer. These fixed cells maintained their morphological integrity, as indicated by light microscope observation. They were

Table 1. Characteristics of fluconazole/itraconazole trailing strains of Candida albicans used in this study.

\begin{tabular}{lcccc}
\hline Code & $\begin{array}{c}\text { Day of isolation } \\
\text { (day/month/year) }\end{array}$ & Site of isolation & \multicolumn{2}{c}{ Patient information } \\
\cline { 4 - 5 } & & Gender & Age \\
\hline $2 \mathrm{~A}$ & $02 / 10 / 2002$ & Blood & Male & - \\
9 & $21 / 03 / 2003$ & Blood & Male & 36 years \\
11 & $30 / 01 / 2003$ & Blood & Male & 46 years \\
$44 \mathrm{~A}$ & $29 / 10 / 2003$ & Gastric washing & Female & 2 years \\
$44 \mathrm{~B}$ & $29 / 10 / 2003$ & Gastric washing & Female & 2 years \\
78 & $12 / 10 / 2004$ & Sputum & Male & 65 years \\
\hline
\end{tabular}

incubated for $1 \mathrm{~h}$ at $25^{\circ} \mathrm{C}$ with a 1:250 dilution of rabbit antiSap1-3 antibody, and then incubated for an additional hour with a 1:200 dilution of fluorescein-isothiocyanate-labeled goat anti-rabbit IgG. For flow cytometry analysis, these cells were examined in an EPICS ELITE flow cytometer (Coulter Electronics, USA) equipped with a 15-mW argon laser emitting at $488 \mathrm{~nm}$. Untreated cells and those treated only with the anti-Sap and secondary antibody were used as controls. Each experimental population was then mapped by using a two-parameter histogram of forward-angle light scatter versus side scatter. The mapped population ( $\mathrm{N}=$ 10,000 ) was then analyzed for log green fluorescence by using a single-parameter histogram (17).

\section{Statistical analysis}

All the experiments, using the same batch culture of each strain, were repeated at least three times and each was performed in triplicate. Representative images of these experiments are shown. Data were analyzed statistically by the Student $t$-test and $P$ values of 0.05 or less were considered to be statistically significant.

\section{Results and Discussion}

Invasive Candida infections continue to cause major problems of morbidity and mortality in a diverse range of debilitated and immunocompromised hosts (21-23). There is concern about the possibility that the empirical use of fluconazole will lead to the emergence of fluconazole-resistant or fluconazole-tolerant Candida isolates. Moreover, the term "trailing" has been used to describe the reduced but persistent growth that some isolates of $C$. albicans and $C$. tropicalis exhibit at drug concentrations above the MIC in broth dilution tests with azole antifungal agents, such as fluconazole and itraconazole (24). This trailing growth can be so great as to make an isolate that appears susceptible after $24 \mathrm{~h}$ of incubation appear resistant at $48 \mathrm{~h}$. In this respect, we recently noticed the trailing phenomenon in our routine susceptibility tests with fluconazole and itraconazole and $C$. albicans clinical isolates (13). The nature of the trailing phenomenon is unknown, as is its contribution to the perception of resistance in vitro and the ultimate possibility of in vivo resistance translation (25). It has been recently suggested that trailing may be due, at least in part, to the ability of $C$. albicans isolates to up-regulate, in response to drug exposure, the transcription of genes encoding the azole drug target, lanosterol demethylase (ERG11), the terbinafine target, squalene epoxidase (ERG1) or the azole and terbinafine efflux transporters (CDR1, CDR2 and MDR1) (25). These same mechanisms, as well as point mutations in ERG11, have been implicated in the development of azole drug resistance in C. albicans $(26,27)$. Furthermore, a simple explanation for the interpretation of the trailing event is that adaptation to a toxic compound can force a longer lag phase in $C$. albicans growth, since adaptation is a time-spanning process. 
C. albicans has developed several virulence mechanisms, which facilitate its invasion of host tissues and the evasion of host defense mechanisms. Virulence factors that contribute to this process are the hydrolytic enzymes, some of which are extracellularly released by the fungus and may play a central role in the pathogenesis of candidiasis. In the present study, we selected six trailing strains of $C$. albicans (Table 1, Figure 1) and a non-trailing ATCC strain (36802) in order to evaluate the activity of aspartyl peptidases, which are well-recognized virulence factors expressed during different phases of $C$. albicans-host interaction, including adhesion, nutrition, proliferation, differentiation, and escape of host immune response (reviewed in Ref. 4). Initially, we investigated the capacity of these seven $C$. albicans strains to grow in vitro in YCB medium containing BSA as the sole nitrogen source. Under these culture conditions, Candida strains might produce extracellular peptidases to degrade BSA molecules in order to obtain free amino acids. By

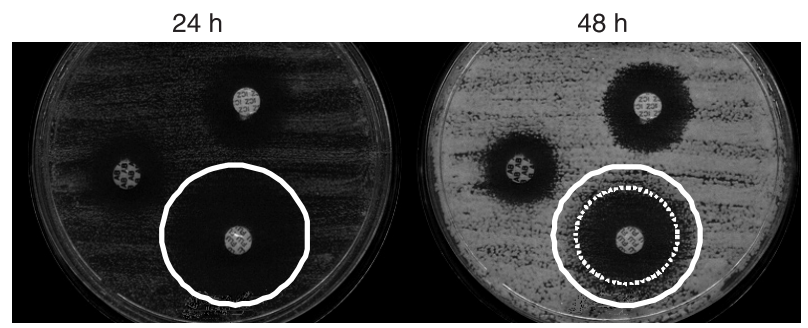

Figure 1. Representative culture of trailing growth observed in Candida albicans. Strain 11 was tested against fluconazole, amphotericin B and 5-fluorocytosine. The white circles show the characteristic trailing growth, which is defined as a resistant MIC after $48 \mathrm{~h}$ (dotted circle) of incubation with triazole antifungal agents but a susceptible MIC after $24 \mathrm{~h}$ (full circle), in the presence of fluconazole after 24 and $48 \mathrm{~h}$ of incubation. The same result was observed when itraconazole and all other trailing strains were tested (data not shown).

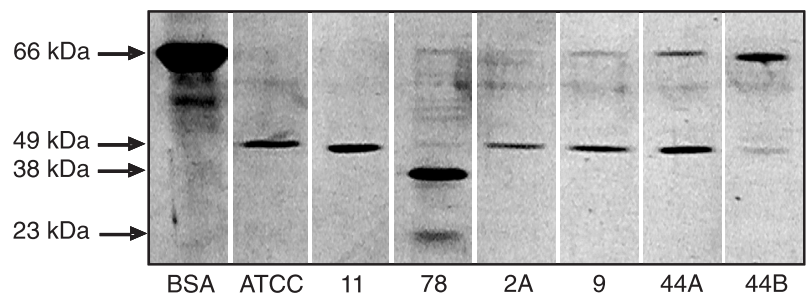

Figure 2. Albumin degradation profiles from different strains of Candida albicans. ATCC strain 36802 and six trailing clinical strains (designated 2A, 9, 11, 44A, 44B, and 78) were grown in YCB medium supplemented with $0.1 \%$ BSA (as the nitrogen source) at $37^{\circ} \mathrm{C}$ under slight agitation. After $48 \mathrm{~h}$ of growth, the cultures were harvested and filtered and the spent culture media were then analyzed by SDS-PAGE to demonstrate the cleavage of soluble BSA. A control in which the culture medium was collected before the yeast inoculation is shown (lane BSA). The gel was silver-stained and the numbers on the left indicate the relative molecular mass of standards, reported in kilodaltons $(\mathrm{kDa})$. means of SDS-PAGE, we showed that all strains were able to hydrolyze soluble BSA at different intensity, generating low molecular mass polypeptides (Figure 2).

BSA cleavage during the growth of $C$. albicans denotes the production of surface and/or released proteolytic activity(ies). In this context, we measured peptidase activity in both cell-free culture supernatants and in intact yeast cells after growth of the trailing strains in YCB-BSA medium for $48 \mathrm{~h}$. Our results demonstrated that yeasts secreted different amounts of pepstatin-sensitive protease activity into the extracellular environment, in which strain 11 was the highest producer, followed by $78,2 \mathrm{~A}$ and ATCC strains (Figure 3A). Strains 9, 44A, and 44B presented the lowest amount of secreted proteolytic activity (Figure $3 A$ ). As

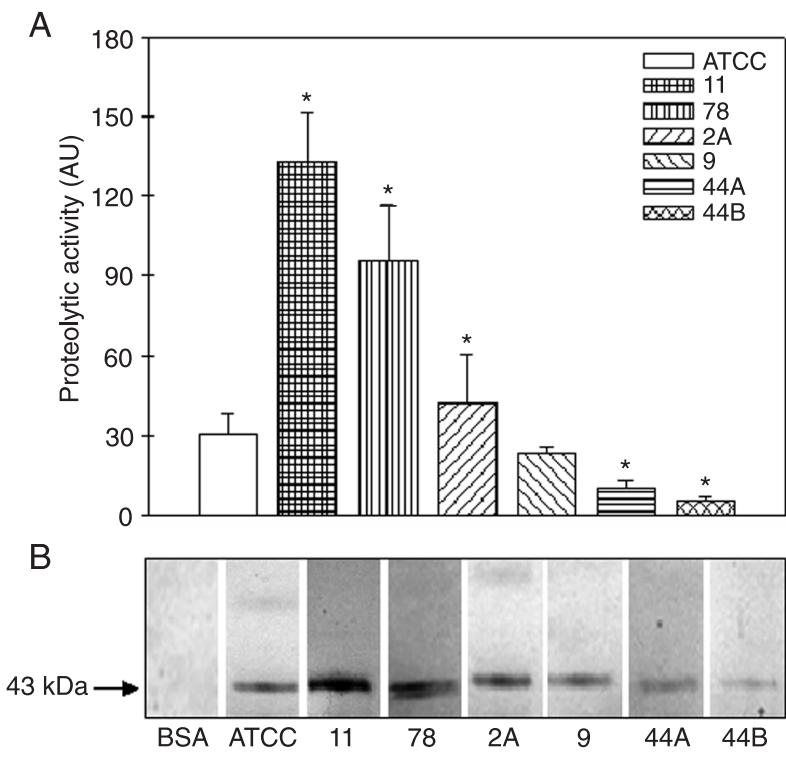

Figure 3. $A$, Measurement of secreted aspartyl peptidase activity on BSA in clinical isolates of Candida albicans. After growth in YCB-BSA medium for $48 \mathrm{~h}$ at $37^{\circ} \mathrm{C}$ under slight agitation, the cultures were harvested and filtered and the spent culture media were then tested to degrade soluble BSA. Each cell-free culture supernatant was supplemented with BSA and $10 \mathrm{mM}$ sodium citrate, $\mathrm{pH} 4.0$, and subsequently incubated at $37^{\circ} \mathrm{C}$ for $2 \mathrm{~h}$. The proteolytic activity was determined as described in Material and Methods and is reported as arbitrary units (AU). The values are reported as the means \pm SEM of three independent experiments, which were performed in triplicate. Asterisks denote trailing clinical isolates that showed a rate of substrate hydrolysis significantly different from the ATCC strain ( ${ }^{*} P<0.05$, Student $t$-test). $B$, Western blotting analysis showing the detection of Sap protein in each $C$. albicans isolate. The proteins in the spent culture media were separated by SDS-PAGE and transferred to a nitrocellulose membrane. A control in which the culture medium was collected before the yeast inoculation is shown (lane BSA). The blot was sequentially incubated with rabbit anti-Sap1-3 polyclonal antibody at 1:1000, peroxidase-conjugated goat anti-rabbit IgG at 1:5000 and then developed with enhanced chemiluminescence reagents. The molecular mass of the reactive polypeptide, reported as kilodaltons $(\mathrm{kDa})$, is given on the left. 
expected, pepstatin A at $10 \mu \mathrm{M}$ completely inhibited the extracellular peptidase activity measured in all C. albicans strains, showing that the peptidases produced belong to the aspartyl peptidase class (data not shown). Anti-Sap1-3 polyclonal antibody recognized, in a Western blotting assay, only one secreted polypeptide of $43 \mathrm{kDa}$ in all $C$. albicans strains (Figure 3B). As previously reported by White and Agabian (16), the 43-kDa component corresponds to the Sap2 isoenzyme. Finally, flow cytometry analysis using the anti-Sap 1-3 antibody detected a comparable level of surface aspartyl peptidase in all trailing strains, except for isolate 9 that presented half the amount of Sap (Figure 4).

The extracellular peptidases of eukaryotic microbial pathogens have attracted the attention of many laboratories because of their potential role in pathogenesis. They facilitate the penetration into the host organism and counteract its defense system. A variety of aspartic peptidases are also produced and secreted by $C$. albicans. Although there are several members of this family, probably individually expressed at various stages of the infection process and playing different roles, Sap2 appears to be the major secretory aspartic peptidase in log-phase Candida cells $(16,28)$. Saps may play an important role in the pathogenicity of candidiasis and their hydrolytic activity probably has a number of possible functions in addition to the simple role of digesting molecules for nutrition $(3,4,29)$. Sap2, as the best-studied member of this group of hydrolytic enzymes, contributes to host tissue invasion by digesting or destroying cell membranes and by degrading host surface molecules. There is also some evidence that Sap2 is able to attack cells and molecules of the host immune system to avoid or resist antimicrobial activity (reviewed in Ref. 4). Furthermore, Sap2 may also contribute to the pathogenesis of inflammatory mucosal lesions in an in vitro model of vaginal candidiasis based on reconstituted human vaginal epithelium, by inducing the up-regulation of epithelial proinflammatory cytokines (29).

Taken together, our results show that the 24-h incubation period of the standard antibiotic sensitivity test is not sufficient for the satisfactory growth of trailing strains. Compounding this problem, C. albicans resistance to antifungal drugs is a major problem in patients receiving prolonged therapy. Strains of C. albicans vary in virulence, but, as is the case for all yeast infections, the balance between transient or commensal colonization and parasitism is dependent on the physiological status of the host. We also demonstrated that trailing clinical isolates of $C$. albicans produced different amounts of both cellular and extracellular aspartyl-like peptidases, suggesting that this phenotypic

\section{References}

1. Olaechea PM, Palomar M, Leon-Gil C, Alvarez-Lerma F, Jorda R, Nolla-Salas J, et al. Economic impact of Candida

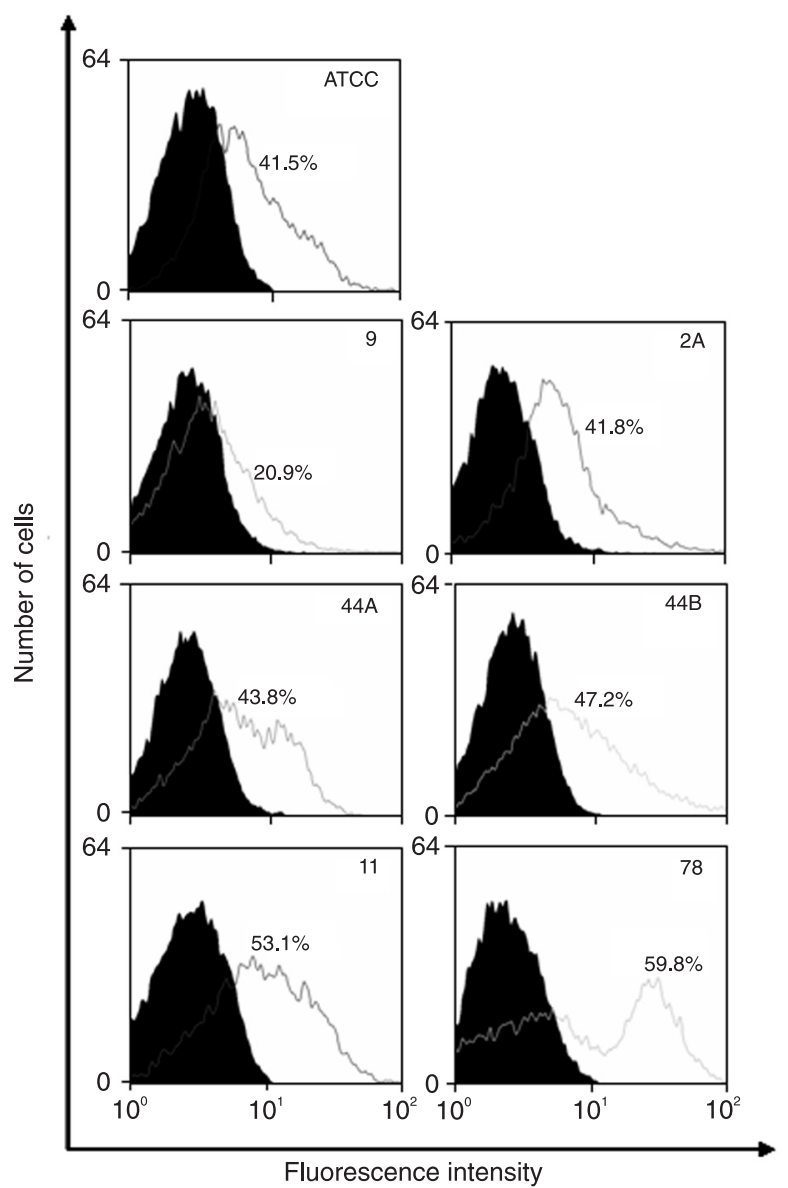

Figure 4. Flow cytometric analysis showing the anti-Sap1-3 antibody binding to Candida albicans. After growth in YCB-BSA medium for $48 \mathrm{~h}$ at $37^{\circ} \mathrm{C}$ under slight agitation, the yeast cells were washed in PBS, fixed in paraformaldehyde and then incubated in the absence (black curves) and in the presence (gray curves) of anti-Sap1-3 antibody at 1:250 dilution for $1 \mathrm{~h}$. Subsequently, the cells were incubated for $1 \mathrm{~h}$ with a 1:200 dilution of FITC-labeled goat anti-rabbit IgG and examined in an EPICS ELITE flow cytometer. The numbers indicate the percentage of cells in each population that was marked with the anti-Sap antibody.

feature did not generate a regular pattern regarding the expression of Sap.

\section{Acknowledgments}

We thank Dr. Marta H. Branquinha for a critical revision of the English text as well as for valuable suggestions about the manuscript. 
2. Ostrosky-Zeichner L, Pappas PG. Invasive candidiasis in the intensive care unit. Crit Care Med 2006; 34: 857-863.

3. Calderone RA, Fonzi WA. Virulence factors of Candida albicans. Trends Microbiol 2001; 9: 327-335.

4. Naglik JR, Challacombe SJ, Hube B. Candida albicans secreted aspartyl proteinases in virulence and pathogenesis. Microbiol Mol Biol Rev 2003; 67: 400-428.

5. Rentz AM, Halpern MT, Bowden R. The impact of candidemia on length of hospital stay, outcome, and overall cost of illness. Clin Infect Dis 1998; 27: 781-788.

6. He X, Tiballi RN, Zarins LT, Bradley SF, Sangeorzan JA, Kauffman CA. Azole resistance in oropharyngeal Candida albicans strains isolated from patients infected with human immunodeficiency virus. Antimicrob Agents Chemother 1994; 38: 2495-2497.

7. Pfaller MA, Lockhart SR, Pujol C, Swails-Wenger JA, Messer $\mathrm{SA}$, Edmond MB, et al. Hospital specificity, region specificity, and fluconazole resistance of Candida albicans bloodstream isolates. J Clin Microbiol 1998; 36: 1518-1529.

8. Barchiesi F, Maracci M, Radi B, Arzeni D, Baldassarri I, Giacometti A, et al. Point prevalence, microbiology and fluconazole susceptibility patterns of yeast isolates colonizing the oral cavities of HIV-infected patients in the era of highly active antiretroviral therapy. J Antimicrob Chemother 2002; 50: 999-1002.

9. Calvet HM, Yeaman MR, Filler SG. Reversible fluconazole resistance in Candida albicans: a potential in vitro model. Antimicrob Agents Chemother 1997; 41: 535-539.

10. Marr KA, Lyons CN, Ha K, Rustad TR, White TC. Inducible azole resistance associated with a heterogeneous phenotype in Candida albicans. Antimicrob Agents Chemother 2001; 45: 52-59.

11. Ollert MW, Wende C, Gorlich M, Mullan-Vogel CG, Borg-von Zepelin M, Vogel CW, et al. Increased expression of Candida albicans secretory proteinase, a putative virulence factor, in isolates from human immunodeficiency virus-positive patients. J Clin Microbiol 1995; 33: 2543-2549.

12. Ozkan S, Kaynak F, Kalkanci A, Abbasoglu U, Kustimur S. Slime production and proteinase activity of Candida species isolated from blood samples and the comparison of these activities with minimum inhibitory concentration values of antifungal agents. Mem Inst Oswaldo Cruz 2005; 100: 319323.

13. Ribeiro MD. Análise comparativa por diferentes metodologias do perfil de susceptibilidade aos antifúngicos de leveduras isoladas de espécimes clínicos. [Master's thesis]: Instituto de Microbiologia Prof. Paulo de Góes, Universidade Federal do Rio de Janeiro, Rio de Janeiro, RJ, Brazil; 2006.

14. Yang YL, Cheng HH, Ho YA, Hsiao CF, Lo HJ. Fluconazole resistance rate of Candida species from different regions and hospital types in Taiwan. J Microbiol Immunol Infect 2003; 36: 187-191.

15. Clinical and Laboratory Standards Institute (formerly NCCLS). Reference method for broth dilution antifungal susceptibility testing of yeasts. Wayne: Approved Standard. M27A; 1997.

16. White TC, Agabian N. Candida albicans secreted aspartyl proteinases: isoenzyme pattern is determined by cell type, and levels are determined by environmental factors. J Bacteriol 1995; 177: 5215-5221.

17. Santos ALS, Carvalho I, Silva BA, Portela MB, Alviano CS, Soares RMA. Secretion of serine peptidase by a clinical strain of Candida albicans: influence of growth conditions and cleavage of human serum proteins and extracellular matrix components. FEMS Immunol Med Microbiol 2006; 46: 209-220.

18. Laemmli UK. Cleavage of structural proteins during the assembly of the head of bacteriophage T4. Nature 1970; 227 : 680-685.

19. Buroker-Kilgore M, Wang KK. A Coomassie brilliant blue G-250-based colorimetric assay for measuring activity of calpain and other proteases. Anal Biochem 1993; 208: 387392.

20. Silva BA, Pinto MR, Soares RMA, Barreto-Bergter E, Santos ALS. Pseudallescheria boydii releases metallopeptidases capable of cleaving several proteinaceous compounds. Res Microbiol 2006; 157: 425-432.

21. Eggimann P, Garbino J, Pittet D. Management of Candida species infections in critically ill patients. Lancet Infect Dis 2003; 3: 772-785.

22. Aperis G, Myriounis N, Spanakis EK, Mylonakis E. Developments in the treatment of candidiasis: more choices and new challenges. Expert Opin Investig Drugs 2006; 15: 13191336.

23. Portela MB, Souza IP, Costa EM, Hagler AN, Soares RMA, Santos ALS. Differential recovery of Candida species from subgingival sites in human immunodeficiency virus-positive and healthy children from Rio de Janeiro, Brazil. J Clin Microbiol 2004; 42: 5925-5927.

24. Lee MK, Williams LE, Warnock DW, Arthington-Skaggs BA. Drug resistance genes and trailing growth in Candida albicans isolates. J Antimicrob Chemother 2004; 53: 217-224.

25. Ostrosky-Zeichner L, Rex JH, Pappas PG, Hamill RJ, Larsen RA, Horowitz HW, et al. Antifungal susceptibility survey of 2,000 bloodstream Candida isolates in the United States. Antimicrob Agents Chemother 2003; 47: 3149-3154.

26. Smith WL, Edlind TD. Histone deacetylase inhibitors enhance Candida albicans sensitivity to azoles and related antifungals: correlation with reduction in CDR and ERG upregulation. Antimicrob Agents Chemother 2002; 46: 35323539.

27. Sanglard D, Odds FC. Resistance of Candida species to antifungal agents: molecular mechanisms and clinical consequences. Lancet Infect Dis 2002; 2: 73-85.

28. Smolenski G, Sullivan PA, Cutfield SM, Cutfield JF. Analysis of secreted aspartic proteinases from Candida albicans: purification and characterization of individual Sap1, Sap2 and Sap3 isoenzymes. Microbiology 1997; 143 (Part 2): 349-356.

29. De Bernardis F, Arancia S, Morelli L, Hube B, Sanglard D, Schafer W, et al. Evidence that members of the secretory aspartyl proteinase gene family, in particular SAP2, are virulence factors for Candida vaginitis. J Infect Dis 1999; 179: 201-208. 